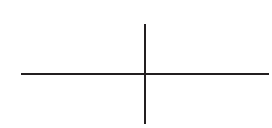

Tohoku Math. J.

57 (2005), 231-246

\title{
A TRANSPLANTATION THEOREM FOR THE HANKEL TRANSFORM ON THE HARDY SPACE
}

\author{
YUICHI KANJIN* \\ (Received August 6, 2003, revised April 5, 2004)
}

\begin{abstract}
The transplantation operators for the Hankel transform are considered and their boundedness on the real Hardy space is established. As its application, we obtain the Hörmander-Mihlin type multiplier theorem for the Hankel transform on the real Hardy space.
\end{abstract}

1. Introduction. The Hankel transform $\mathcal{H}_{\mu} f$ of order $\mu$ of a function $f$ on the open half line $(0, \infty)$ is defined by

$$
\mathcal{H}_{\mu} f(y)=\int_{0}^{\infty} f(t) \sqrt{y t} J_{\mu}(y t) d t, \quad y>0,
$$

where $J_{\mu}$ is the Bessel function of the first kind of order $\mu$. The Bessel functions with $\mu=$ $-1 / 2$ and $\mu=1 / 2$ are

$$
J_{-1 / 2}(z)=\sqrt{\frac{2}{\pi z}} \cos z, \quad J_{1 / 2}(z)=\sqrt{\frac{2}{\pi z}} \sin z
$$

and the Hankel transforms $\mathcal{H}_{-1 / 2} f$ and $\mathcal{H}_{1 / 2} f$ are the cosine and sine transforms:

$$
\mathcal{H}_{-1 / 2} f(y)=\sqrt{\frac{2}{\pi}} \int_{0}^{\infty} f(t) \cos y t d t, \quad \mathcal{H}_{1 / 2} f(y)=\sqrt{\frac{2}{\pi}} \int_{0}^{\infty} f(t) \sin y t d t .
$$

It is known that for $\mu \geq-1 / 2, \mathcal{H}_{\mu}$ is an isometry on $L^{2}(0, \infty)$ (Parseval's theorem for the Hankel transform) and $\mathcal{H}_{\mu} \mathcal{H}_{\mu}=I$ (The inversion formula for the Hankel transform), and

$$
\int_{0}^{\infty} f(x) g(x) d x=\int_{0}^{\infty} \mathcal{H}_{\mu} f(x) \mathcal{H}_{\mu} g(x) d x
$$

for $f, g \in L^{2}(0, \infty)$ (Plancherel's theorem for the Hankel transform), where $I$ is the identity operator and $L^{2}(0, \infty)$ is the Lebesgue space of functions on $(0, \infty)$ such that $\|f\|_{2}=$ $\left(\int_{0}^{\infty}|f(x)|^{2} d x\right)^{1 / 2}<\infty$.

We shall consider the composite

$$
\mathcal{T}_{\mu}^{v}=\mathcal{H}_{\mu} \mathcal{H}_{\nu}
$$

2000 Mathematics Subject Classification. Primary 42C10; Secondary 42B30, 33C45.

Key words and phrases. Hankel transform, transplantation theorem, Hardy space.

*Partly supported by the Grants-in-Aid for Scientific Research (C), Japan Society for the Promotion of Science. 


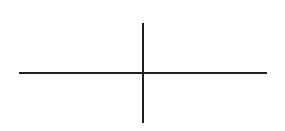

which is an isometry on $L^{2}(0, \infty)$ for $\mu, v \geq-1 / 2$. For $f \in L^{1}(0, \infty)$ with $\mathcal{H}_{v} f \in$ $L^{1}(0, \infty), \mathcal{T}_{\mu}^{\nu} f$ has the integral representation

$$
\mathcal{T}_{\mu}^{v} f(x)=\int_{0}^{\infty} \int_{0}^{\infty} f(t) \sqrt{y t} J_{v}(y t) d t \sqrt{x y} J_{\mu}(x y) d y, \quad x>0 .
$$

We call $\mathcal{T}_{\mu}^{\nu}$ the transplantation operator from $v$ to $\mu$. The aim of this paper is to prove that the transplantation operators $\mathcal{T}_{\mu}^{v}$ are bounded on the real Hardy space. As an application, we shall obtain the Hörmander-Mihlin type multiplier theorem for the Hankel transform on the real Hardy space.

The main tools of our proofs are the atomic decomposition and the molecular characterization of the real Hardy space, and Schindler's integral representation [13] of $\mathcal{T}_{\mu}^{\nu}$. We recall the representation here. Let $T_{\mu, \nu}$ be an operator defined by

$$
\begin{gathered}
T_{\mu, \nu} f(x)=\lim _{\delta \rightarrow+0} \int_{|x-y|>\delta} f(y) \tilde{I}_{\mu, v}(x, y) d y+k(\mu, v) f(x), \\
k(\mu, \nu)=\cos ((\mu-v) \pi / 2),
\end{gathered}
$$

where

$$
\begin{aligned}
\tilde{I}_{\mu, v}(x, y) & \\
& =K_{\mu, v} \sqrt{x y}\left(\frac{y}{x}\right)^{v} \frac{1}{x^{2}-y^{2}} F\left(\frac{v-\mu}{2}, \frac{\mu+v}{2} ; v+1 ; \frac{y^{2}}{x^{2}}\right) \\
& =2^{-1} K_{\mu, v}\left(\frac{y}{x}\right)^{v+1 / 2}\left(\frac{1}{x-y}+\frac{1}{x+y}\right) F\left(\frac{v-\mu}{2}, \frac{\mu+v}{2} ; v+1 ; \frac{y^{2}}{x^{2}}\right), \\
K_{\mu, v} & =\frac{2 \Gamma((\mu+v+2) / 2)}{\Gamma(v+1) \Gamma((\mu-v) / 2)}
\end{aligned}
$$

for $0<y<x$, and

$$
\tilde{I}_{\mu, v}(x, y)=\tilde{I}_{v, \mu}(y, x)
$$

for $y>x>0$. Here, $F(\alpha, \beta ; \gamma ; z)$ is the hypergeometric function, that is,

$$
F(\alpha, \beta ; \gamma ; z)=\sum_{k=0}^{\infty} \frac{(\alpha)_{k}(\beta)_{k}}{(\gamma)_{k} k !} z^{k}, \quad|z|<1,
$$

where $(\lambda)_{0}=1,(\lambda)_{k}=\lambda(\lambda+1) \cdots(\lambda+k-1), k \geq 1$. If $\mu=v+2 k$ and $k=0,1,2, \ldots$, then $\tilde{I}_{\mu, v}(x, y)=0$ for $y>x>0$. If $k=0,-1,-2, \ldots$, then $\tilde{I}_{\mu, v}(x, y)=0$ for $x>y>0$. In these cases, $\tilde{I}_{\mu, v}(x, y)$ have more elementary forms (see [13]). Schindler proved that if $\mu, v \geq-1 / 2$, then the following (A) and (B) hold:

(A) For $f \in C_{c}^{\infty}(0, \infty), \mathcal{T}_{\mu}^{\nu} f(x)=T_{\mu, \nu} f(x)$ a.e. $x>0$, where $C_{c}^{\infty}(0, \infty)$ is the space of infinitely differentiable functions of compact support in $(0, \infty)$;

(B) Let $1<p<\infty$ and $-1 / p<\alpha<1-1 / p$. If $\int_{0}^{\infty}|f(x)|^{p} x^{\alpha p} d x<\infty$, then the value $T_{\mu, \nu} f(x)$ exists for a.e. $x>0$, and

$$
\int_{0}^{\infty}\left|T_{\mu, \nu} f(x)\right|^{p} x^{\alpha p} d x \leq C \int_{0}^{\infty}|f(x)|^{p} x^{\alpha p} d x
$$




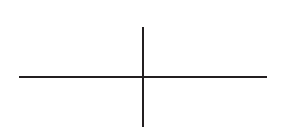

with a constant $C$ independent of $f$.

Guy [6] proved that the operators $\mathcal{T}_{\mu}^{\nu}$, initially defined on $L^{2}(0, \infty)$, are extendable to bounded operators on the $L^{p}$-spaces, $1<p<\infty$, and this is the first of the transplantation theorem for classical expansions. Schindler [13] showed a refined version of Guy's result by getting the explicit formula of $\mathcal{T}_{\mu}^{\nu}$ as we recalled above.

To consider the transplantation operators $\mathcal{T}_{\mu}^{v}$ for the case $p=1$ is our problem, and the main result of this paper is that the operators $\mathcal{T}_{\mu}^{\nu}$ are bounded on the real Hardy space, which gives us the Hörmander-Mihlin type multiplier theorem for the Hankel transform on the real Hardy space.

There are transplantation theorems for other orthogonal expansions. Askey and Wainger [2] gave a transplantation theorem for the ultraspherical series, and Askey [1] generalized their theorem to the Jacobi series. Some transplantation theorems are in Gilbert [5] and in Muckenhoupt [12]. The Laguerre series case is in Kanjin [7]. Miyachi [10] and [11] quite recently obtained a transplantation theorem for the Jacobi series in weighted Hardy spaces.

The author would like to thank the referee for careful reading of the paper and comments.

2. Results. Let $H^{1}(\boldsymbol{R})$ be the real Hardy space, that is, the space of the boundary functions $f(x)=\Re F(x)$ of the real parts $\Re F(z)$ of functions $F(z)$ in the Hardy space $H^{1}\left(\boldsymbol{R}_{+}^{2}\right)=\left\{F(z)\right.$; analytic in $\boldsymbol{R}_{+}^{2}$ and $\left.\|F\|_{H^{1}\left(\boldsymbol{R}_{+}^{2}\right)}=\sup _{t>0} \int_{-\infty}^{\infty}|F(x+i t)| d x<\infty\right\}$ on the upper half plane $\boldsymbol{R}_{+}^{2}=\{z=x+i t ; t>0\}$, with the norm $\|f\|_{H^{1}(\boldsymbol{R})}=\|F\|_{H^{1}\left(\boldsymbol{R}_{+}^{2}\right)}$.

We shall work on the space $H^{1}(0, \infty)$ defined by

$$
H^{1}(0, \infty)=\left\{\left.h\right|_{(0, \infty)} ; h \in H^{1}(\boldsymbol{R}), \operatorname{supp} h \subset[0, \infty)\right\},
$$

where $[0, \infty)$ is the closed half line, and we endow the space with the norm $\|f\|_{H^{1}(0, \infty)}=$ $\|h\|_{H^{1}(\boldsymbol{R})}$, where $h \in H^{1}(\boldsymbol{R}), \operatorname{supp} h \subset[0, \infty)$ and $f=\left.h\right|_{(0, \infty)}$. We remark that

$$
H^{1}(0, \infty)=\left\{\left.h\right|_{(0, \infty)} ; h \in H^{1}(\boldsymbol{R}), \text { even }\right\}
$$

and $c_{1}\|h\|_{H^{1}(\boldsymbol{R})} \leq\|f\|_{H^{1}(0, \infty)} \leq c_{2}\|h\|_{H^{1}(\boldsymbol{R})}$ with positive constants $c_{1}$ and $c_{2}$, where $f=$ $\left.h\right|_{(0, \infty)}$ and $h \in H^{1}(\boldsymbol{R})$ is even. This fact is in [4, Chapter III, Lemma 7.40].

Our theorem is as follows:

THEOREM. (i) Let $\mu \geq-1 / 2$ and $v>-1 / 2$. Then $\mathcal{T}_{\mu}^{v}$, initially defined on $H^{1}(0, \infty) \cap L^{2}(0, \infty)$, is uniquely extended to a bounded operator on $H^{1}(0, \infty)$, and if we still denote it by $\mathcal{T}_{\mu}^{\nu}$, then

$$
\left\|\mathcal{T}_{\mu}^{v} f\right\|_{H^{1}(0, \infty)} \leq C\|f\|_{H^{1}(0, \infty)}
$$

for $f \in H^{1}(0, \infty)$ with a constant $C$ depending only on $\mu$ and $\nu$.

(ii) If $\mu \geq-1 / 2$, then $\mathcal{T}_{\mu}^{-1 / 2}$ is uniquely extended to a bounded operator from $H^{1}(0, \infty)$ to $L^{1}(0, \infty)$, that is,

$$
\left\|\mathcal{T}_{\mu}^{-1 / 2} f\right\|_{L^{1}(0, \infty)} \leq C\|f\|_{H^{1}(0, \infty)}
$$

for $f \in H^{1}(0, \infty)$ with a constant $C$ depending only on $\mu$ and $\nu$. 


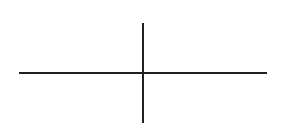

As an application of our theorem we deal with the Hörmander-Mihlin type multiplier theorem for the Hankel transform. Let $\mu \geq-1 / 2$ and $\phi \in L^{\infty}(0, \infty)$. We define a Hankel multiplier operator $\mathcal{M}_{\phi}^{\mu}$ with multiplier $\phi$ by

$$
\mathcal{M}_{\phi}^{\mu} f=\mathcal{H}_{\mu}\left(\phi \mathcal{H}_{\mu}(f)\right)
$$

for $f \in L^{2}(0, \infty)$. Since $\mathcal{H}_{\mu}$ is an isometry on $L^{2}(0, \infty)$, the multiplier operator $\mathcal{M}_{\phi}^{\mu}$ is a bounded operator on $L^{2}(0, \infty)$ with the operator norm $\|\phi\|_{\infty}$. We also define a Fourier multiplier operator $\mathcal{M}_{m}$ with multiplier $m \in L^{\infty}(\boldsymbol{R})$ by

$$
\mathcal{M}_{m} h=\mathcal{F}^{-1}(m \mathcal{F}(h))
$$

for $h \in L^{2}(\boldsymbol{R})$, where $\mathcal{F}$ and $\mathcal{F}^{-1}$ are the Fourier transform and the inverse Fourier transform, respectively:

$$
\mathcal{F}(h)(\xi)=\frac{1}{\sqrt{2 \pi}} \int_{\boldsymbol{R}} h(x) e^{-i x \xi} d x, \quad \mathcal{F}^{-1}(g)(x)=\frac{1}{\sqrt{2 \pi}} \int_{\boldsymbol{R}} g(\xi) e^{i x \xi} d \xi .
$$

The Hörmander-Mihlin multiplier theorem for $H^{1}(\boldsymbol{R})$ says that, if $m$ with $\|m\|_{L^{\infty}(\boldsymbol{R})} \leq A$ satisfies the condition

$$
\left(\frac{1}{R} \int_{R<|\xi| \leq 2 R}\left|\frac{d m(\xi)}{d \xi}\right|^{2} d \xi\right)^{1 / 2} \leq A R^{-1}
$$

for $R>0$, where $A$ is independent of $R$, then the Fourier multiplier operator $\mathcal{M}_{m}$, initially defined on $H^{1}(\boldsymbol{R}) \cap L^{2}(\boldsymbol{R})$, is uniquely extended to a bounded operator on $H^{1}(\boldsymbol{R})$. If we still denote it by $\mathcal{M}_{m}$, then $\left\|\mathcal{M}_{m} h\right\|_{H^{1}(\boldsymbol{R})} \leq C A\|h\|_{H^{1}(\boldsymbol{R})}$ for $h \in H^{1}(\boldsymbol{R})$ with $C$ independent of $h$ and $m$ (cf. [4, Chapter III, Theorem 7.30]). We may refer to [14, Chapter IV, §3, §6] and [4, Chapter II, Theorem 6.3] for the $L^{p}$-space case.

COROllary. Let $\mu \geq-1 / 2$. Suppose that $\phi$ with $\|\phi\|_{L^{\infty}(0, \infty)} \leq$ A satisfies the condition

$$
\left(\frac{1}{R} \int_{R<y \leq 2 R}\left|\frac{d \phi(y)}{d y}\right|^{2} d y\right)^{1 / 2} \leq A R^{-1}
$$

for $R>0$, where $A$ is independent of $R$. Then the Hankel multiplier operator $\mathcal{M}_{\phi}^{\mu}$, initially defined on $H^{1}(0, \infty) \cap L^{2}(0, \infty)$, is uniquely extended to a bounded operator from $H^{1}(0, \infty)$ to $L^{1}(0, \infty)$. If we also denote it by $\mathcal{M}_{\phi}^{\mu}$, then

$$
\left\|\mathcal{M}_{\phi}^{\mu} f\right\|_{L^{1}(0, \infty)} \leq C A\|f\|_{H^{1}(0, \infty)}
$$

for $f \in H^{1}(0, \infty)$ with $C$ independent of $f$ and $\phi$.

The corollary is deduced from the theorem as follows. Let $\phi \in L^{\infty}(0, \infty)$ satisfy the condition (4), and let $f \in H^{1}(0, \infty) \cap C_{c}^{\infty}(0, \infty)$. We extend $\phi$ and $f$ to the functions on $\boldsymbol{R}$ as even functions, and we denote them by $\phi_{e}$ and $f_{e}$. Since the function $\phi_{e}$ satisfies the condition (3), the Fourier multiplier operator $\mathcal{M}_{\phi_{e}}$ is a bounded operator on $H^{1}(\boldsymbol{R})$. Since $\mathcal{H}_{-1 / 2} f(y)=\mathcal{F} f_{e}(y), \quad y>0$, we see that $\mathcal{M}_{\phi_{e}} f_{e}(x)=\mathcal{M}_{\phi}^{-1 / 2} f(x), \quad x>0$. Further, 


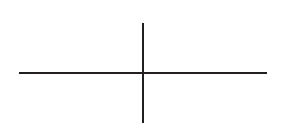

TRANSPLANTATION THEOREM FOR HANKEL TRANSFORM

$\mathcal{M}_{\phi_{e}} f_{e}$ is an even function. Thus, $\mathcal{M}_{\phi}^{-1 / 2}$ has a unique bounded extension on $H^{1}(0, \infty)$. The inequality $\|g\|_{L^{1}(0, \infty)} \leq\|g\|_{H^{1}(0, \infty)}$ holds, and so $\mathcal{M}_{\phi}^{-1 / 2}$ is uniquely extended to a bounded operator from $H^{1}(0, \infty)$ to $L^{1}(0, \infty)$. Let $\mu>-1 / 2$. It follows from the theorem that $\mathcal{T}_{-1 / 2}^{\mu}$ is a bounded operator on $H^{1}(0, \infty)$ and $\mathcal{T}_{\mu}^{-1 / 2}$ is a bounded operator from $H^{1}(0, \infty)$ to $L^{1}(0, \infty)$. Therefore, the identity $\mathcal{M}_{\phi}^{\mu}=\mathcal{T}_{\mu}^{-1 / 2} \mathcal{M}_{\phi}^{-1 / 2} \mathcal{T}_{-1 / 2}^{\mu}$ on $L^{2}(0, \infty)$ implies the corollary.

Remark. Let $\mu>-1 / 2$. Assume that $\mathcal{M}_{\phi}^{\mu}$ is a bounded operator on $H^{1}(0, \infty)$. Then, $\phi=0$ if we assume additionally that $\phi$ satisfies $\phi=\mathcal{H}_{-1 / 2} \Phi$ for some $\Phi \in L^{1}(0, \infty)$. For, we first note that $\mathcal{M}_{\phi}^{1 / 2}$ is a bounded operator on $H^{1}(0, \infty)$ by the identity $\mathcal{M}_{\phi}^{1 / 2}=$ $\mathcal{T}_{1 / 2}^{\mu} \mathcal{M}_{\phi}^{\mu} \mathcal{T}_{\mu}^{1 / 2}$ and the theorem. Let $f \in H^{1}(0, \infty) \cap C_{c}^{\infty}(0, \infty)$. Since $\mathcal{M}_{\phi}^{1 / 2} f \in H^{1}(0, \infty)$, $\mathcal{M}_{\phi}^{1 / 2} f$ has the vanishing mean property:

$$
\int_{0}^{\infty} \mathcal{M}_{\phi}^{1 / 2} f(x) d x=0 .
$$

We extend $\phi$ and $\Phi$ to the even functions on $\boldsymbol{R}$, and denote them by $\phi_{e}$ and $\Phi_{e}$. We note that $\phi_{e}=\mathcal{F} \Phi_{e}$. Further, we extend $f$ to the odd function on $\boldsymbol{R}$, which is denoted by $f_{o}$. Since $-i \mathcal{H}_{1 / 2} f(y)=\mathcal{F} f_{o}(y), \quad y>0$, we see that $\mathcal{M}_{\phi}^{1 / 2} f(x)=\mathcal{M}_{\phi_{e}} f_{o}(x), x>0$. The identity $\mathcal{M}_{\phi_{e}} f_{o}=\Phi_{e} * f_{o}$ holds. Therefore, we have

$$
\begin{aligned}
0 & =\int_{0}^{\infty} \int_{-\infty}^{\infty} \Phi_{e}(y) f_{o}(x-y) d y d x \\
& =\int_{-\infty}^{\infty} \Phi_{e}(y) \int_{0}^{\infty} f_{o}(x-y) d x d y \\
& =\int_{0}^{\infty} \Phi_{e}(y) \int_{-y}^{\infty} f_{o}(u) d u d y+\int_{-\infty}^{0} \Phi_{e}(y) \int_{-y}^{\infty} f_{o}(u) d u d y \\
& =-2 \int_{0}^{\infty} \Phi(y) \int_{0}^{y} f(u) d u d y=-2 \int_{0}^{\infty} f(u) \int_{u}^{\infty} \Phi(y) d y d u,
\end{aligned}
$$

that is, $\int_{0}^{\infty} f(u) \int_{u}^{\infty} \Phi(y) d y d u=0$ for all $f \in H^{1}(0, \infty) \cap C_{c}^{\infty}(0, \infty)$, which implies that $\int_{u}^{\infty} \Phi(y) d y$ is a constant function in $u$, and so $\Phi(y)=0$ for a.e. $y>0$. We conclude $\phi=0$.

We conjecture that without the additional condition the above statement holds, that is, if $\mathcal{M}_{\phi}^{\mu}$ with $\phi \in L^{\infty}(0, \infty)$ is a bounded operator on $H^{1}(0, \infty)$, then $\phi$ is constant, where $\mu>-1 / 2$.

The theorem will be proved in the next section. The atomic decomposition and the molecular characterization of the real Hardy space will play important roles in our proof. A real-valued function $a$ is called an atom centered at $c$ if (i) $a(x)$ is supported in an interval [c-h/2,c+h/2], (ii) $\|a\|_{2} \leq h^{-1 / 2}$, and (iii) $\int_{\boldsymbol{R}} a(x) d x=0$. The space $H^{1}(\boldsymbol{R})$ is characterized in terms of atoms: $f \in H^{1}(\boldsymbol{R})$ if and only if $f=\sum_{j=0}^{\infty} \lambda_{j} a_{j}$, where each $a_{j}$ is an atom and $\sum_{j=0}^{\infty}\left|\lambda_{j}\right|<\infty$. Further, the $H^{1}$-norm $\|f\|_{H^{1}(\boldsymbol{R})}$ is equivalent to $\inf \sum_{j=0}^{\infty}\left|\lambda_{j}\right|$, 


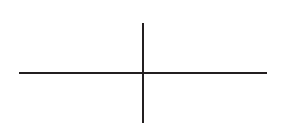

the infimum being taken over all decompositions, and the series $\sum_{j=0}^{\infty} \lambda_{j} a_{j}$ converges in $H^{1}$-norm.

We deal with the functions $f \in H^{1}(0, \infty)$. These functions are also characterized as follows (see [4, Chapter III, Lemma 7.40]): $f \in H^{1}(0, \infty)$ if and only if $f=\sum_{j=0}^{\infty} \lambda_{j} a_{j}$, where each $a_{j}$ is an atom with supp $a_{j} \subset[0, \infty)$ and $\sum_{j=0}^{\infty}\left|\lambda_{j}\right|<\infty$. Moreover, the norm $\|f\|_{H^{1}[0, \infty)}$ is equivalent to inf $\sum_{j=0}^{\infty}\left|\lambda_{j}\right|$, the infimum being taken over all such decompositions. By this decomposition, we see that $H^{1}(0, \infty) \cap L^{2}(0, \infty)$ is dense in $H^{1}(0, \infty)$.

We call a real-valued function $M$ a molecule centered at $c$ if $M$ satisfies the following conditions: (i)

$$
N(M)=\|M\|_{L^{2}(\boldsymbol{R})}^{1 / 2}\||\cdot-c| M\|_{L^{2}(\boldsymbol{R})}^{1 / 2}<\infty ;
$$

(ii) $\int_{\boldsymbol{R}} M(x) d x=0$. We call $N(M)$ the molecular norm of $M(x)$. The molecular characterization asserts that if $f=\sum_{j} M_{j}$ with molecules $M_{j}$ and $\sum_{j} N\left(M_{j}\right)<\infty$, then $f \in H^{1}(\boldsymbol{R})$ and $\|f\|_{H^{1}(\boldsymbol{R})} \leq C \sum_{j} N\left(M_{j}\right)$ with an absolute constant $C$. For the atomic decomposition and the molecular characterization, we may refer to $[4, \mathrm{III}]$.

3. Proofs. The theorem will be proved by the following two lemmas.

LEMMA 1. If $v>-1 / 2$, then $\mathcal{T}_{v}^{v+2}$ and $\mathcal{T}_{v+2}^{v}$ are uniquely extended to bounded operators on $H^{1}(0, \infty)$, that is,

$$
\begin{aligned}
\left\|\mathcal{T}_{v}^{v+2} f\right\|_{H^{1}(0, \infty)} & \leq C\|f\|_{H^{1}(0, \infty)} \\
\left\|\mathcal{T}_{v+2}^{v} f\right\|_{H^{1}(0, \infty)} & \leq C\|f\|_{H^{1}(0, \infty)}
\end{aligned}
$$

for $f \in H^{1}(0, \infty)$ with a constant $C$ depending only on $v$.

LEMMA 2. (i) If $\mu \geq-1 / 2$ and $v \geq 1 / 2$, then $\mathcal{T}_{\mu}^{v}$ is uniquely extended to a bounded operator on $H^{1}(0, \infty)$, that is,

$$
\left\|\mathcal{T}_{\mu}^{v} f\right\|_{H^{1}(0, \infty)} \leq C\|f\|_{H^{1}(0, \infty)}
$$

for $f \in H^{1}(0, \infty)$ with a constant $C$ depending only on $\mu$ and $\nu$.

(ii) If $\mu \geq-1 / 2$, then $\mathcal{T}_{\mu}^{-1 / 2}$ is uniquely extended to a bounded operator from $H^{1}(0, \infty)$ to $L^{1}(0, \infty)$, that is,

$$
\left\|\mathcal{T}_{\mu}^{-1 / 2} f\right\|_{L^{1}(0, \infty)} \leq C\|f\|_{H^{1}(0, \infty)}
$$

for $f \in H^{1}(0, \infty)$ with a constant $C$ depending only on $\mu$.

We see here that the theorem is deduced from these lemmas. We first note that the identity $\mathcal{T}_{\mu}^{\tau} \mathcal{T}_{\tau}^{v}=\mathcal{T}_{\mu}^{v}$ on $L^{2}(0, \infty)$ holds since

$$
\mathcal{T}_{\mu}^{\tau} \mathcal{T}_{\tau}^{v}=\mathcal{H}_{\mu} \mathcal{H}_{\tau} \mathcal{H}_{\tau} \mathcal{H}_{\nu}=\mathcal{H}_{\mu} \mathcal{H}_{\nu}=\mathcal{T}_{\mu}^{\nu}
$$

Let us prove the part (i) of the theorem. Let $\mu \geq-1 / 2$ and $v>-1 / 2$. It follows from Lemma 1 that $\mathcal{T}_{v+2}^{v}$, initially defined on $H^{1}(0, \infty) \cap L^{2}(0, \infty)$, is uniquely extended to a bounded operator on $H^{1}(0, \infty)$. Since $v+2 \geq 1 / 2$, it follows from the part (i) of Lemma 2 that the operator $\mathcal{T}_{\mu}^{\nu+2}$ is uniquely extended to a bounded operator on $H^{1}(0, \infty)$. Because 


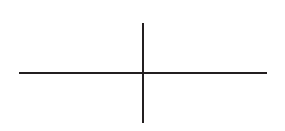

TRANSPLANTATION THEOREM FOR HANKEL TRANSFORM

of the fact $\mathcal{T}_{\mu}^{v}=\mathcal{T}_{\mu}^{\nu+2} \mathcal{T}_{v+2}^{v}$ on $H^{1}(0, \infty) \cap L^{2}(0, \infty)$, we see that $\mathcal{T}_{\mu}^{v}$ has a unique bounded extension on $H^{1}(0, \infty)$. The part (ii) of the theorem is the part (ii) of Lemma 2 itself.

We turn to the proof of Lemma 1 . Let $v>-1 / 2$, and put

$$
U^{(v)} f(x)=\int_{x}^{\infty}\left(\frac{x}{t}\right)^{v+1 / 2} f(t) \frac{d t}{t}, \quad S^{(v)} f(x)=\frac{1}{x} \int_{0}^{x}\left(\frac{t}{x}\right)^{v+1 / 2} f(t) d t
$$

for $x>0$. Then, we see that

$$
\mathcal{T}_{v}^{v+2} f=2(v+1) U^{(v)} f-f, \quad \mathcal{T}_{v+2}^{v} f=2(v+1) S^{(v)} f-f
$$

for $f \in L^{2}(0, \infty)$ by [13, p. 383, line 5 from below and p. 381, line 8 from below]. In [8, Proposition], we proved that $U^{(v)}$ and $S^{(v)}$ are extended to bounded operators on $H^{1}(0, \infty)$ for $v>-1 / 2$, and thus, $\mathcal{T}_{v}^{v+2}$ and $\mathcal{T}_{v+2}^{v}$ have the same boundedness, which is Lemma 1.

Lemma 2 will be reduced to the following Lemma 3 and Lemma 4.

Lemma 3. Assume that $(\mu, v)$ satisfies $\mu \geq-1 / 2, v \geq 1 / 2$ or $\mu \geq-1 / 2, v=-1 / 2$. Let $a$ be an atom centered at $c$ with supp $a \subset[0, \infty)$, and we regard $\mathcal{T}_{\mu}^{v} a$ as $\mathcal{T}_{\mu}^{v} a(x)=0$ for $x \leq 0$. Then, there exists a constant $C$ depending only on $\mu$ and $v$ such that

$$
N\left(\mathcal{T}_{\mu}^{v} a\right)=\left\|\mathcal{T}_{\mu}^{v} a\right\|_{2}^{1 / 2}\left\||\cdot-c| \mathcal{T}_{\mu}^{v} a\right\|_{2}^{1 / 2} \leq C .
$$

Lemma 4. Let $\mu \geq-1 / 2$ and $v \geq 1 / 2$. Then, $\int_{0}^{\infty} \mathcal{T}_{\mu}^{v} a(x) d x=0$ for every atom $a$ with $\operatorname{supp} a \subset[0, \infty)$.

We show first that Lemma 2 is obtained by Lemma 3 and Lemma 4. Let $f \in H^{1}(0, \infty) \cap$ $L^{2}(0, \infty)$. Let $f=\sum_{j=0}^{\infty} \lambda_{j} a_{j}$ be an atomic decomposition of $f$ such that $\sum_{j=0}^{\infty}\left|\lambda_{j}\right| \leq$ $C\|f\|_{H^{1}(0, \infty)}$, where $C$ is independent of $f$. To prove Lemma 2, we shall first show that

$$
\mathcal{T}_{\mu}^{v} f(x)=\sum_{j=0}^{\infty} \lambda_{j} \mathcal{T}_{\mu}^{v} a_{j}(x) \quad \text { a.e. } x>0
$$

for $\mu \geq-1 / 2, v \geq 1 / 2$ or $\mu \geq-1 / 2, v=-1 / 2$. Let $g \in C_{c}^{\infty}(0, \infty)$. Then we have

$$
\int_{0}^{\infty} \mathcal{T}_{\mu}^{\nu} f(x) g(x) d x=\int_{0}^{\infty} \mathcal{H}_{\mu} \mathcal{H}_{\nu} f(x) g(x) d x=\int_{0}^{\infty} f(x) \mathcal{H}_{\nu} \mathcal{H}_{\mu} g(x) d x
$$

by Plancherel's theorem and the inversion formula. The inequality $\left|\mathcal{H}_{\nu} \mathcal{H}_{\mu} g(x)\right| \leq$ $C\left\|\mathcal{H}_{\mu} g\right\|_{L^{1}(0, \infty)}$ holds, and $\left\|\mathcal{H}_{\mu} g\right\|_{L^{1}(0, \infty)}<\infty$ since $g \in C_{c}^{\infty}(0, \infty)$. For every atom $a_{j}$, we have $\left\|a_{j}\right\|_{L^{1}(0, \infty)} \leq 1$. Thus we have

$$
\begin{aligned}
\int_{0}^{\infty} f(x) \mathcal{H}_{\nu} \mathcal{H}_{\mu} g(x) d x & =\int_{0}^{\infty} \sum_{j=0}^{\infty} \lambda_{j} a_{j}(x) \mathcal{H}_{\nu} \mathcal{H}_{\mu} g(x) d x \\
& =\sum_{j=0}^{\infty} \lambda_{j} \int_{0}^{\infty} a_{j}(x) \mathcal{H}_{\nu} \mathcal{H}_{\mu} g(x) d x \\
& =\sum_{j=0}^{\infty} \lambda_{j} \int_{0}^{\infty} \mathcal{H}_{\mu} \mathcal{H}_{\nu} a_{j}(x) g(x) d x
\end{aligned}
$$




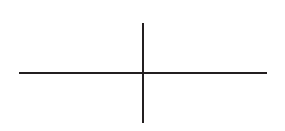

We remark that the inequality $\|\psi\|_{L^{1}(0, \infty)} \leq 2^{3 / 2} N(\psi)$ holds (cf. [4, Chapter III, Lemma 7.11]). It follows from Lemma 3 that

$$
\left\|\mathcal{H}_{\mu} \mathcal{H}_{\nu} a_{j}\right\|_{L^{1}(0, \infty)}=\left\|\mathcal{T}_{\mu}^{v} a_{j}\right\|_{L^{1}(0, \infty)} \leq C N\left(\mathcal{T}_{\mu}^{v} a_{j}\right) \leq C
$$

Here and below, $C$ denotes a positive constant which may differ at each different occurrence. Thus, the last sum is equal to

$$
\int_{0}^{\infty} \sum_{j=0}^{\infty} \lambda_{j} \mathcal{H}_{\mu} \mathcal{H}_{\nu} a_{j}(x) g(x) d x
$$

which leads to

$$
\int_{0}^{\infty} \mathcal{T}_{\mu}^{v} f(x) g(x) d x=\int_{0}^{\infty} \sum_{j=0}^{\infty} \lambda_{j} \mathcal{T}_{\mu}^{v} a_{j}(x) g(x) d x
$$

for all $g \in C_{c}^{\infty}(0, \infty)$, and we get (6).

Because of (6), we have

$$
\begin{aligned}
\left\|\mathcal{T}_{\mu}^{v} f\right\|_{H^{1}(0, \infty)} & \leq C \sum_{j=0}^{\infty} N\left(\lambda_{j} \mathcal{T}_{\mu}^{v} a_{j}\right) \leq C \sum_{j=0}^{\infty}\left|\lambda_{j}\right| N\left(\mathcal{T}_{\mu}^{v} a_{j}\right) \\
& \leq C \sum_{j=0}^{\infty}\left|\lambda_{j}\right| \leq C\|f\|_{H^{1}(0, \infty)}
\end{aligned}
$$

for $\mu \geq-1 / 2, v \geq 1 / 2$ by Lemma 3 , Lemma 4 and the molecular characterization. If $\mu \geq-1 / 2, v=-1 / 2$, then

$$
\begin{aligned}
\left\|\mathcal{T}_{\mu}^{v} f\right\|_{L^{1}(0, \infty)} & \leq \sum_{j=0}^{\infty}\left|\lambda_{j}\right|\left\|\mathcal{T}_{\mu}^{v} a_{j}\right\|_{L^{1}(0, \infty)} \leq C \sum_{j=0}^{\infty}\left|\lambda_{j}\right| N\left(\mathcal{T}_{\mu}^{v} a_{j}\right) \\
& \leq C \sum_{j=0}^{\infty}\left|\lambda_{j}\right| \leq C\|f\|_{H^{1}(0, \infty)} .
\end{aligned}
$$

These inequalities allow us to use the standard density argument, and we obtain Lemma 2.

We now come to the proofs of Lemma 3 and Lemma 4.

Proof of Lemma 3. Let $a$ be an atom centered at $c$ with supp $a \subset[0, \infty)$. Let $Q=[c-h / 2, c+h / 2] \subset[0, \infty)$ be the smallest interval containing supp $a$. Since $\mathcal{T}_{\mu}^{v}$ is an isometry on $L^{2}(0, \infty)$, it follows that

$$
\left\|\mathcal{T}_{\mu}^{v} a\right\|_{2}=\|a\|_{2} \leq h^{-1 / 2} .
$$

To prove (5), it is enough to show that $\left\||\cdot-c| \mathcal{T}_{\mu}^{v} a\right\|_{2} \leq C h^{1 / 2}$. We put $\tilde{Q}=[c-h, c+h]$. We write

$$
\begin{aligned}
\left\||\cdot-c| \mathcal{T}_{\mu}^{v} a\right\|_{2}^{2} & =\left\{\int_{(0, \infty) \cap \tilde{Q}}+\int_{(0, \infty) \backslash \tilde{Q}}\right\}|x-c|^{2}\left|\mathcal{T}_{\mu}^{v} a(x)\right|^{2} d x \\
& =V_{1}+V_{2}, \quad \text { say } .
\end{aligned}
$$




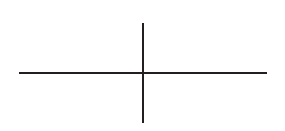

TRANSPLANTATION THEOREM FOR HANKEL TRANSFORM

For $V_{1}$, we have by (7),

$$
V_{1} \leq h^{2}\left\|\mathcal{T}_{\mu}^{v} a\right\|_{2}^{2} \leq h
$$

An essential part of the proof is to show $V_{2} \leq C h$. By Schindler's results (A) and (B), we see that $\mathcal{T}_{\mu}^{v}=T_{\mu, v}$ on $L^{2}(0, \infty)$. Thus, Schindler's integral representation (1) leads us to

$$
\mathcal{T}_{\mu}^{v} a(x)=\lim _{\delta \rightarrow+0} \int_{|x-y|>\delta} a(y) \tilde{I}(x, y) d y+k(\mu, v) a(x) \quad \text { a.e. } x>0,
$$

where we put $\tilde{I}(x, y)=\tilde{I}_{\mu, v}(x, y)$ for simplicity. For $x \in(0, \infty) \backslash \tilde{Q}$, we have

$$
\mathcal{T}_{\mu}^{v} a(x)=\int_{Q} a(y) \tilde{I}(x, y) d y,
$$

and thus,

$$
V_{2}=\int_{(0, \infty) \backslash \tilde{Q}}|x-c|^{2}\left|\int_{Q} a(y) \tilde{I}(x, y) d y\right|^{2} d x
$$

The Taylor expansion of $\tilde{I}(x, y)$ in $y$ at $c$ and the cancellation property of atoms imply

$$
\int_{Q} a(y) \tilde{I}(x, y) d y=\int_{Q} a(y) \frac{\partial \tilde{I}}{\partial y}(x, c+\theta(y-c))(y-c) d y, \quad 0<\theta<1 .
$$

If we show

(8) $\left|\frac{\partial \tilde{I}}{\partial y}(x, \xi)\right| \leq \frac{C}{|x-c|^{2}}, \quad \xi=c+\theta(y-c), 0<\theta<1, y \in Q, x \in(0, \infty) \backslash \tilde{Q}$

with $C$ depending only on $\mu$ and $\nu$, then

$$
\begin{aligned}
\left|\int_{Q} a(y) \tilde{I}(x, y) d y\right| & \leq \frac{C}{|x-c|^{2}} \int_{Q}|a(y)||y-c| d y \\
& \leq \frac{C}{|x-c|^{2}}\|a\|_{2} h^{3 / 2} \leq \frac{C}{|x-c|^{2}} h
\end{aligned}
$$

which leads to the desired inequality

$$
V_{2} \leq C h^{2} \int_{(0, \infty) \backslash \tilde{Q}} \frac{d x}{|x-c|^{2}} \leq C h .
$$

The rest of the proof is devoted to proving (8). We divide the matter into two cases; Case I: $c+h<x$ and $y \in Q$; Case II: $0<x<c-h$ and $y \in Q$.

We begin with Case I. Since $0<y<x$, it follows from (2) that

$$
\frac{\partial \tilde{I}}{\partial y}(x, y)=2^{-1} K_{\mu, v}\left\{W_{1}^{+}(x, y)+W_{2}^{+}(x, y)+W_{3}^{+}(x, y)\right\},
$$




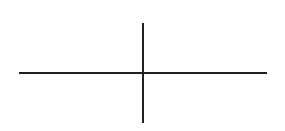

where

$$
\begin{aligned}
& W_{1}^{+}(x, y)=\left(v+\frac{1}{2}\right)\left(\frac{y}{x}\right)^{v-1 / 2} \frac{1}{x}\left(\frac{1}{x-y}+\frac{1}{x+y}\right) F\left(\frac{v-\mu}{2}, \frac{v+\mu}{2} ; v+1 ; \frac{y^{2}}{x^{2}}\right), \\
& W_{2}^{+}(x, y)=\left(\frac{y}{x}\right)^{v+1 / 2}\left(\frac{1}{(x-y)^{2}}+\frac{-1}{(x+y)^{2}}\right) F\left(\frac{v-\mu}{2}, \frac{v+\mu}{2} ; v+1 ; \frac{y^{2}}{x^{2}}\right),
\end{aligned}
$$

and

$$
\begin{aligned}
& W_{3}^{+}(x, y)=\left(\frac{y}{x}\right)^{v+1 / 2}\left(\frac{1}{x-y}+\frac{1}{x+y}\right) \frac{\partial}{\partial y}\left\{F\left(\frac{v-\mu}{2}, \frac{v+\mu}{2} ; v+1 ; \frac{y^{2}}{x^{2}}\right)\right\} \\
& =\frac{v^{2}-\mu^{2}}{2(v+1)}\left(\frac{y}{x}\right)^{v+3 / 2} \frac{1}{x}\left(\frac{1}{x-y}+\frac{1}{x+y}\right) F\left(\frac{v-\mu+2}{2}, \frac{v+\mu+2}{2} ; v+2 ; \frac{y^{2}}{x^{2}}\right)
\end{aligned}
$$

from the formula $(d / d z) F(\alpha, \beta ; \gamma ; z)=(\alpha \beta / \gamma) F(\alpha+1, \beta+1 ; \gamma+1 ; z)$. We shall show

$$
\left|W_{j}^{+}(x, \xi)\right| \leq \frac{C}{|x-c|^{2}}, \quad j=1,2,3
$$

with $C$ depending only on $\mu$ and $\nu$.

Since

$$
\lim _{z \rightarrow 1-} F(\alpha, \beta ; \gamma ; z)=\frac{\Gamma(\gamma) \Gamma(\gamma-\alpha-\beta)}{\Gamma(\gamma-\alpha) \Gamma(\gamma-\beta)}
$$

for $\Re(\gamma-\alpha-\beta)>0$ (cf. [9, (9.3.4)]), it follows from $v+1-(v-\mu) / 2-(\nu+\mu) / 2=1$ and $\xi<x$ that

$$
\left|F\left(\frac{v-\mu}{2}, \frac{v+\mu}{2} ; v+1 ; \frac{\xi^{2}}{x^{2}}\right)\right| \leq C
$$

for $0<y<x$ with a constant $C$ depending only on $\mu, \nu$. We see that $(\xi / x)^{\nu-1 / 2} \leq 1$ for $0<y<x$ when $v-1 / 2 \geq 0$, and that

$$
\left|W_{1}^{+}(x, \xi)\right| \leq C \frac{1}{x}\left(\frac{1}{|x-\xi|}+\frac{1}{x+\xi}\right) .
$$

Since $\xi \in Q$ and $c+h<x$, it follows that $|x-\xi| \geq|x-c| / 2$. Also, $x+\xi>x>|x-c|$. These imply the inequality (9) with $j=1$. We note that the term $W_{1}^{+}$does not appear in $\partial \tilde{I} / \partial y$ when $v=-1 / 2$.

For $W_{2}^{+}(x, \xi)$, in a similar way, we have

$$
\left|W_{2}^{+}(x, \xi)\right| \leq C\left(\frac{1}{|x-\xi|^{2}}+\frac{1}{(x+\xi)^{2}}\right) \leq \frac{C}{|x-c|^{2}}
$$

for $v \geq-1 / 2$, which is the inequality(9) with $j=2$.

To estimate $W_{3}^{+}(x, \xi)$, we use the formula (cf. [9, (9.2.6)]):

$$
\gamma(1-z) F(\alpha, \beta ; \gamma ; z)-\gamma F(\alpha-1, \beta ; \gamma ; z)+(\gamma-\beta) z F(\alpha, \beta ; \gamma+1 ; z)=0 \text {. }
$$




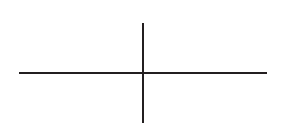

TRANSPLANTATION THEOREM FOR HANKEL TRANSFORM

The substitution $\alpha=(v-\mu+2) / 2, \beta=(v+\mu+2) / 2, \gamma=v+2, z=y^{2} / x^{2}$ gives

$$
F\left(\frac{v-\mu+2}{2}, \frac{v+\mu+2}{2} ; \nu+2 ; \frac{y^{2}}{x^{2}}\right)=\frac{x^{2}}{x^{2}-y^{2}} F_{1}-\frac{v-\mu+2}{2(v+2)} \frac{y^{2}}{x^{2}-y^{2}} F_{2},
$$

where

$$
\begin{aligned}
& F_{1}=F\left(\frac{v-\mu}{2}, \frac{v+\mu+2}{2} ; v+2 ; \frac{y^{2}}{x^{2}}\right) \\
& F_{2}=F\left(\frac{v-\mu+2}{2}, \frac{v+\mu+2}{2} ; \nu+3 ; \frac{y^{2}}{x^{2}}\right) .
\end{aligned}
$$

This implies

$$
\begin{aligned}
W_{3}^{+}(x, \xi)= & \left.c_{\mu, \nu}\left(\frac{\xi}{x}\right)^{v+3 / 2}\left(\frac{1}{x-\xi}+\frac{1}{x+\xi}\right)^{2} F_{1}\right|_{y=\xi} \\
& +\left.c_{\mu, \nu}^{\prime}\left(\frac{\xi}{x}\right)^{v+5 / 2}\left(\frac{1}{(x-\xi)^{2}}-\frac{1}{(x+\xi)^{2}}\right) F_{2}\right|_{y=\xi},
\end{aligned}
$$

where $c_{\mu, \nu}$ and $c_{\mu, \nu}^{\prime}$ are some constants depending only on $\mu$ and $\nu$. We note that $\left|F_{1}\right|,\left|F_{2}\right| \leq$ $C$ for $0<y<x$ since $v+2-(v-\mu) / 2-(v+\mu+2) / 2=1$ and $v+3-(v-\mu+2) / 2-$ $(v+\mu+2) / 2=1$. Thus, in the same way as in the above cases, we have the inequality (9) with $j=3$, which completes Case I.

We turn to Case II. It follows from $0<x<y$ that

$$
\begin{gathered}
\frac{\partial \tilde{I}}{\partial y}(x, y)=2^{-1} K_{v, \mu}\left\{W_{1}^{-}(x, y)+W_{2}^{-}(x, y)+W_{3}^{-}(x, y)\right\}, \\
W_{1}^{-}(x, y)=-\left(\mu+\frac{1}{2}\right)\left(\frac{x}{y}\right)^{\mu+1 / 2} \frac{1}{y}\left(\frac{1}{y-x}+\frac{1}{y+x}\right) F\left(\frac{\mu-v}{2}, \frac{v+\mu}{2} ; \mu+1 ; \frac{x^{2}}{y^{2}}\right), \\
W_{2}^{-}(x, y)=\left(\frac{x}{y}\right)^{\mu+1 / 2}\left(\frac{-1}{(y-x)^{2}}+\frac{-1}{(y+x)^{2}}\right) F\left(\frac{\mu-v}{2}, \frac{v+\mu}{2} ; \mu+1 ; \frac{x^{2}}{y^{2}}\right)
\end{gathered}
$$

and

$$
\begin{aligned}
W_{3}^{-}(x, y)= & \left(\frac{x}{y}\right)^{\mu+1 / 2}\left(\frac{1}{y-x}+\frac{1}{y+x}\right) \frac{\partial}{\partial y}\left\{F\left(\frac{\mu-v}{2}, \frac{v+\mu}{2} ; \mu+1 ; \frac{x^{2}}{y^{2}}\right)\right\} \\
=- & \frac{\mu^{2}-v^{2}}{2(\mu+1)}\left(\frac{x}{y}\right)^{\mu+5 / 2} \frac{1}{y}\left(\frac{1}{y-x}+\frac{1}{y+x}\right) \\
& \quad \times F\left(\frac{\mu-v+2}{2}, \frac{v+\mu+2}{2} ; \mu+2 ; \frac{x^{2}}{y^{2}}\right) \\
=- & c_{v, \mu}\left(\frac{x}{y}\right)^{\mu+5 / 2}\left(\frac{1}{y-x}+\frac{1}{y+x}\right)^{2} F_{3} \\
& \quad-c_{\nu, \mu}^{\prime}\left(\frac{x}{y}\right)^{\mu+7 / 2}\left(\frac{1}{(y-x)^{2}}-\frac{1}{(y+x)^{2}}\right) F_{4},
\end{aligned}
$$




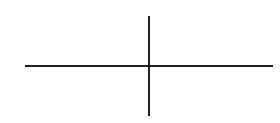

where

$$
\begin{aligned}
& F_{3}=F\left(\frac{\mu-v}{2}, \frac{v+\mu+2}{2} ; \mu+2 ; \frac{x^{2}}{y^{2}}\right), \\
& F_{4}=F\left(\frac{\mu-v+2}{2}, \frac{v+\mu+2}{2} ; \mu+3 ; \frac{x^{2}}{y^{2}}\right) .
\end{aligned}
$$

Since $0<h<c$, it follows that $\xi \geq c-h / 2 \geq c / 2 \geq|x-c| / 2$, which implies $1 /(x+\xi) \leq$ $1 / \xi \leq 2 /|x-c|$. This inequality and $1 /|x-\xi| \leq 2 /|x-c|$ allow us to follow the lines of the proof of Case I if $\mu \geq-1 / 2$, and get the inequality (8) in Case II. We complete the proof of Lemma 3.

PRoOF OF Lemma 4. Let $a$ be an atom with supp $a \subset[0, \infty)$. It follows from Lemma 3 and the inequality $\left\|\mathcal{T}_{\mu}^{v} a\right\|_{L^{1}(0, \infty)} \leq 2^{3 / 2} N\left(\mathcal{T}_{\mu}^{v} a\right)$ that $\mathcal{T}_{\mu}^{v} a$ is integrable for $\mu \geq-1 / 2, v \geq$ $1 / 2$ or $\mu \geq-1 / 2, \nu=-1 / 2$. Thus, for these $\mu$, $\nu$, we have

$$
\int_{0}^{\infty} \mathcal{T}_{\mu}^{v} a(x) d x=\lim _{\varepsilon \rightarrow+0} \int_{0}^{\infty} e^{-\varepsilon x^{2}} \mathcal{T}_{\mu}^{v} a(x) d x .
$$

By the fact

$$
\mathcal{T}_{\mu}^{v} a(x)=\lim _{M \rightarrow \infty} \int_{0}^{M} \mathcal{H}_{\nu} a(y) \sqrt{x y} J_{\mu}(x y) d y
$$

in $L^{2}(0, \infty)$, we have

$$
\begin{aligned}
\int_{0}^{\infty} \mathcal{T}_{\mu}^{v} a(x) d x & =\lim _{\varepsilon \rightarrow+0} \lim _{M \rightarrow \infty} \int_{0}^{\infty} e^{-\varepsilon x^{2}} \int_{0}^{M} \mathcal{H}_{\nu} a(y) \sqrt{x y} J_{\mu}(x y) d y d x \\
& =\lim _{\varepsilon \rightarrow+0} \lim _{M \rightarrow \infty} \int_{0}^{\infty} e^{-\varepsilon x^{2}} \int_{0}^{M} \int_{0}^{\infty} a(t) \sqrt{y t} J_{\nu}(y t) d t \sqrt{x y} J_{\mu}(x y) d y d x
\end{aligned}
$$

Since $\left|\sqrt{z} J_{\alpha}(z)\right| \leq C, z>0$ for $\alpha \geq-1 / 2$ and $e^{-\varepsilon x^{2}} a(t)$ is integrable in $(x, y, t)$ on $(0, \infty) \times$ $(0, M) \times(0, \infty)$, it follows that

$$
\int_{0}^{\infty} \mathcal{T}_{\mu}^{v} a(x) d x=\lim _{\varepsilon \rightarrow+0} \lim _{M \rightarrow \infty} \int_{0}^{\infty} a(t) B_{M}^{(\varepsilon)}(t) d t
$$

for $\mu, v \geq-1 / 2$, where

$$
\begin{aligned}
B_{M}^{(\varepsilon)}(t) & =\int_{0}^{M} D_{t}^{(\varepsilon)}(y) d y \\
D_{t}^{(\varepsilon)}(y) & =\int_{0}^{\infty} e^{-\varepsilon x^{2}} \sqrt{x y} J_{\mu}(x y) d x \sqrt{t y} J_{v}(t y) .
\end{aligned}
$$

To prove $\int_{0}^{\infty} \mathcal{T}_{\mu}^{v} a(x) d x=0$, we shall show the following:

(I) Let $t>0,0<\varepsilon<1$ and $1<M$. If $\mu>-3 / 2, \neq-1$ and $v>-1 / 2$, then $\left|B_{M}^{(\varepsilon)}(t)\right| \leq C$, where $C$ depends only on $\mu$ and $\nu$.

(II) For every $t>0, \lim _{\varepsilon \rightarrow+0} \lim _{M \rightarrow \infty} B_{M}^{(\varepsilon)}(t)=C_{\mu, \nu}$, where

$$
C_{\mu, v}=\frac{\Gamma(\mu / 2+4 / 3) \Gamma(v / 2+1 / 4)}{\Gamma(\mu / 2+1 / 4) \Gamma(v / 2+3 / 4)}
$$




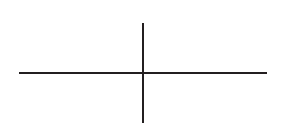

TRANSPLANTATION THEOREM FOR HANKEL TRANSFORM

when $\mu>-3 / 2, \neq-1$ and $v>-1 / 2$.

If we show (I) and (II), then by the Lebesgue dominated convergence theorem we shall get

$$
\int_{0}^{\infty} \mathcal{T}_{\mu}^{v} a(x) d x=\int_{0}^{\infty} a(t) \lim _{\varepsilon \rightarrow+0} \lim _{M \rightarrow \infty} B_{M}^{(\varepsilon)}(t) d t=C_{\mu, \nu} \int_{0}^{\infty} a(t) d t=0
$$

for $\mu \geq-1 / 2$ and $\nu \geq 1 / 2$, and the proof of Lemma 4 will be completed.

Let us prove (I) and (II). We shall use the formula (cf. [15, 13.3(3), p. 394])

$$
\begin{aligned}
\int_{0}^{\infty} e^{-\varepsilon x^{2}} & \sqrt{x y} J_{\mu}(x y) d x \\
& =\frac{y^{\mu+1 / 2} \Gamma((\mu+3 / 2) / 2)}{2^{\mu+1} \varepsilon^{(\mu+3 / 2) / 2} \Gamma(\mu+1)} e^{-y^{2} /(4 \varepsilon)} \Phi\left((\mu+1 / 2) / 2 ; \mu+1 ; y^{2} /(4 \varepsilon)\right),
\end{aligned}
$$

where $\mu>-3 / 2$ and $\Phi(\alpha ; \gamma ; z)$ is Kummer's confluent hypergeometric series defined by $\Phi(\alpha ; \gamma ; z)=\sum_{k=0}^{\infty}\left[(\alpha)_{k} /(\gamma)_{k}\right]\left[z^{k} / k !\right]$ for $z, \alpha, \gamma \in C, \quad \gamma \neq 0,-1,-2, \ldots$ Since $\Phi(\alpha ; \gamma ; z)$ is an entire function of $z$, it follows that for $0<y \leq 2 \sqrt{\varepsilon}$,

$$
\left|\int_{0}^{\infty} e^{-\varepsilon x^{2}} \sqrt{x y} J_{\mu}(x y) d x\right| \leq C \varepsilon^{-\mu / 2-3 / 4} y^{\mu+1 / 2},
$$

when $\mu>-3 / 2$. The asymptotic formula (cf. [3, 6.13.1(3), Vol. 1, p. 278])

$$
\Phi(\alpha ; \gamma ; z)=\frac{\Gamma(\gamma)}{\Gamma(\alpha)} e^{z} z^{\alpha-\gamma}\left[1+O\left(|z|^{-1}\right)\right], \quad \Re z \rightarrow \infty, \quad \gamma \neq 0,-1,-2, \ldots
$$

gives, for $2 \sqrt{\varepsilon} \leq y$,

$$
\int_{0}^{\infty} e^{-\varepsilon x^{2}} \sqrt{x y} J_{\mu}(x y) d x=C_{\mu} y^{-1}+R_{\varepsilon}(y), \quad\left|R_{\varepsilon}(y)\right| \leq C \varepsilon y^{-3},
$$

if $\mu>-3 / 2, \neq-1$, where $C$ depends only on $\mu$ and $C_{\mu}=2^{1 / 2} \Gamma((\mu+3 / 2) / 2) / \Gamma((\mu+$ 1/2)/2).

Let $t>0,0<\varepsilon<1$ and $1<M$. We divide the integral $B_{M}^{(\varepsilon)}(t)=\int_{0}^{M} D_{t}^{(\varepsilon)}(y) d y$ into two parts:

$$
B_{M}^{(\varepsilon)}(t)=\left\{\int_{0}^{2 \sqrt{\varepsilon}}+\int_{2 \sqrt{\varepsilon}}^{M}\right\} D_{t}^{(\varepsilon)}(y) d y .
$$

We begin with estimating the integral $\int_{0}^{2 \sqrt{\varepsilon}} D_{t}^{(\varepsilon)}(y) d y$. By (10) and $\left|\sqrt{z} J_{v}(z)\right| \leq C, z>0$ for $v \geq-1 / 2$, we have

$$
\left|\int_{0}^{2 \sqrt{\varepsilon}} D_{t}^{(\varepsilon)}(y) d y\right| \leq \int_{0}^{2 \sqrt{\varepsilon}}\left|D_{t}^{(\varepsilon)}(y)\right| d y \leq C \varepsilon^{-\mu / 2-3 / 4} \int_{0}^{2 \sqrt{\varepsilon}} y^{\mu+1 / 2} d y=C
$$

for $\mu>-3 / 2$ and $v \geq-1 / 2$, where $C$ depends only on $\mu$ and $\nu$. Let $t>0$ be fixed and let $\varepsilon>0$ be sufficiently small so that $2 \sqrt{\varepsilon}<1 / t$. By (10) and the fact $J_{v}(z)=O\left(z^{v}\right)(z \rightarrow 0)$ 


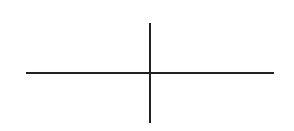

for $v \neq-1,-2, \ldots$, we have

$$
\left|\int_{0}^{2 \sqrt{\varepsilon}} D_{t}^{(\varepsilon)}(y) d y\right| \leq C \varepsilon^{-\mu / 2-3 / 4} \int_{0}^{2 \sqrt{\varepsilon}} y^{\mu+1 / 2}(t y)^{\nu+1 / 2} d y=C t^{\nu+1 / 2} \varepsilon^{\nu / 2+1 / 4} .
$$

Thus, for every $t>0$, we have

$$
\lim _{\varepsilon \rightarrow 0} \int_{0}^{2 \sqrt{\varepsilon}} D_{t}^{(\varepsilon)}(y) d y=0
$$

when $\mu>-3 / 2$ and $\nu>-1 / 2$.

We next estimate the integral $\int_{2 \sqrt{\varepsilon}}^{M} D_{t}^{(\varepsilon)}(y) d y$. By (11), we have

$$
\int_{2 \sqrt{\varepsilon}}^{M} D_{t}^{(\varepsilon)}(y) d y=C_{\mu} U_{1}+U_{2}
$$

where

$$
U_{1}=\int_{2 \sqrt{\varepsilon}}^{M} \sqrt{t y} J_{v}(t y) y^{-1} d y, \quad U_{2}=\int_{2 \sqrt{\varepsilon}}^{M} \sqrt{t y} J_{v}(t y) R_{\varepsilon}(y) d y
$$

for $\mu>-3 / 2, \neq-1$.

The integral $U_{2}$ is estimated by (11) and $\left|\sqrt{z} J_{v}(z)\right| \leq C, z>0$ for $v \geq-1 / 2$. We have

$$
\left|U_{2}\right| \leq C \varepsilon \int_{2 \sqrt{\varepsilon}}^{\infty} y^{-3} d y \leq C
$$

for $\mu>-3 / 2, \neq-1$ and $v \geq-1 / 2$. Let $t>0$ be fixed, and let $\varepsilon>0$ be sufficiently small and $M$ be sufficiently large so that $2 \sqrt{\varepsilon}<1 / t<M$. We divide the integral as follows:

$$
U_{2}=\left\{\int_{2 \sqrt{\varepsilon}}^{1 / t}+\int_{1 / t}^{M}\right\} \sqrt{t y} J_{v}(t y) R_{\varepsilon}(y) d y=U_{2}^{1}+U_{2}^{2}, \quad \text { say . }
$$

By the fact $J_{v}(z)=O\left(z^{v}\right)(z \rightarrow 0)$ for $v \neq-1,-2, \ldots$, we have

$$
\begin{aligned}
\left|U_{2}^{1}\right| \leq & C \int_{2 \sqrt{\varepsilon}}^{1 / t}(t y)^{\nu+1 / 2} \varepsilon y^{-3} d y \\
& \leq\left\{\begin{array}{l}
C \int_{0}^{1 / t}(t y)^{v+1 / 2} \varepsilon y^{-3} d y \leq C t^{2} \varepsilon \quad(v>3 / 2), \\
C \int_{2 \sqrt{\varepsilon}}^{1 / t} t^{2} \varepsilon y^{-1} d y \leq C t^{2} \varepsilon(|\log t|+\log (1 / \varepsilon)) \quad(v=3 / 2), \\
C \int_{2 \sqrt{\varepsilon}}^{\infty}(t y)^{v+1 / 2} \varepsilon y^{-3} d y \leq C t^{\nu+1 / 2} \varepsilon^{(v+1 / 2) / 2} \quad(v<3 / 2)
\end{array}\right.
\end{aligned}
$$

for $\mu>-3 / 2, \neq-1$ and $v \neq-1,-2, \ldots$ It follows from the fact $\sqrt{z} J_{\alpha}(z)=O(1)(z \rightarrow$ $\infty)$ that

$$
\left|U_{2}^{2}\right| \leq C \int_{1 / t}^{\infty} \varepsilon y^{-3} d y \leq C t^{2} \varepsilon
$$




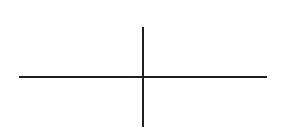

TRANSPLANTATION THEOREM FOR HANKEL TRANSFORM

Therefore, we have

$$
\lim _{\varepsilon \rightarrow 0} \lim _{M \rightarrow \infty} U_{2}=0
$$

for $\mu>-3 / 2, \neq-1$ and $\nu>-1 / 2$.

We turn to estimating $U_{1}$. We first deal with the case $2 \sqrt{\varepsilon} \leq 1 / t \leq M$ and divide the integral:

$$
U_{1}=\left\{\int_{2 \sqrt{\varepsilon}}^{1 / t}+\int_{1 / t}^{M}\right\} \sqrt{t y} J_{v}(t y) y^{-1} d y=U_{1}^{1}+U_{1}^{2}, \quad \text { say } .
$$

By the fact $J_{v}(z)=O\left(z^{v}\right)(z \rightarrow 0)$ for $v \neq-1,-2, \ldots$, we have $\left|U_{1}^{1}\right| \leq$ $C \int_{0}^{1 / t}(t y)^{v+1 / 2} y^{-1} d y$. Thus, if $v>-1 / 2$, then $\left|U_{1}^{1}\right| \leq C$. Let us evaluate $U_{1}^{2}$. The Bessel function satisfies $(d / d z) z^{-\alpha} J_{\alpha}(z)=-z^{-\alpha} J_{\alpha+1}(z)$. This and integration by parts lead to

$$
\begin{aligned}
U_{1}^{2}= & \left(-t^{\nu-3 / 2}\right) \int_{1 / t}^{M} y^{\nu-3 / 2} \frac{d}{d y}\left((t y)^{-v+1} J_{\nu-1}(t y)\right) d y \\
= & \left(-t^{\nu-3 / 2}\right)\left[y^{\nu-3 / 2}(t y)^{-v+1} J_{\nu-1}(t y)\right]_{1 / t}^{M} \\
& -\left(-t^{\nu-3 / 2}\right) \int_{1 / t}^{M}(t y)^{-v+1} J_{\nu-1}(t y) \frac{d}{d y}\left(y^{\nu-3 / 2}\right) d y=U_{1}^{2,1}+U_{1}^{2,2}, \quad \text { say . }
\end{aligned}
$$

The first term $U_{1}^{2,1}=-(t M)^{-1 / 2} J_{v-1}(t M)+J_{v-1}(1)$ satisfies $\left|U_{1}^{2,1}\right| \leq C$ since $\sqrt{z} J_{\alpha}(z)=$ $O(1)(z \rightarrow \infty)$ and $1 \leq t M$. The second term

$$
U_{1}^{2,2}=\left(v-\frac{3}{2}\right) \frac{1}{t} \int_{1 / t}^{M} y^{-2} \sqrt{t y} J_{v-1}(t y) d y
$$

is evaluated as follows: $\left|U_{1}^{2,2}\right| \leq C t^{-1} \int_{1 / t}^{\infty} y^{-2} d y \leq C$. Thus, we have $\left|U_{1}^{2}\right| \leq C$ and then $\left|U_{1}\right| \leq C$ in the case $2 \sqrt{\varepsilon} \leq 1 / t \leq M$. In the case $1 / t<2 \sqrt{\varepsilon}$, we have $\left|U_{1}\right| \leq C$ in the same way as in the estimation of $U_{1}^{2}$, and in the case $M<1 / t$, we also have $\left|U_{1}\right| \leq C$ in the same way as in the estimation of $U_{1}^{1}$. Therefore, these and (14) imply

$$
\left|\int_{2 \sqrt{\varepsilon}}^{M} D_{t}^{(\varepsilon)}(y) d y\right| \leq C
$$

for $\mu>-3 / 2, \neq-1$ and $v>-1 / 2$.

Combining (12) and (16), we have (I). The statement (II) is proved as follows: By (13) and (15), we have

$$
\begin{aligned}
\lim _{\varepsilon \rightarrow+0} \lim _{M \rightarrow \infty} B_{M}^{(\varepsilon)}(t) & =C_{\mu} \lim _{\varepsilon \rightarrow+0} \lim _{M \rightarrow \infty} U_{1}=C_{\mu} \int_{0}^{\infty} \sqrt{t y} J_{v}(t y) y^{-1} d y \\
& =C_{\mu} \int_{0}^{\infty} J_{v}(u) u^{-1 / 2} d u=C_{\mu, v}
\end{aligned}
$$

for every $t>0$ when $\mu>-3 / 2, \neq-1$ and $v>-1 / 2$. We here used $\int_{0}^{\infty} J_{v}(u) u^{-1 / 2} d u=$ $\Gamma(v / 2+1 / 4) /(\Gamma(v / 2+3 / 4) \sqrt{2})$ for $v>-1 / 2$. 


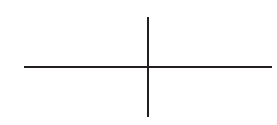

\section{REFERENCES}

[1] R. Askey, A transplantation theorem for Jacobi series, Illinois J. Math. 13 (1969), 583-590.

[2] R. ASKey AND S. WAINGER, A transplantation theorem between ultraspherical series, Illinois J. Math. 10 (1966), 322-344.

[3] A. Erdélyi, W. Magnus, F. Oberhettinger and F. G. Tricomi, Higher transcendental functions, 3 volumes, McGraw-Hill Book Company, Inc., New York-Toronto-London, 1953.

[4] J. Garcia-Cuerva and J. L. Rubio de Francia, Weighted norm inequalities and related topics, NorthHolland Math. Stud. 116, Notas de Matemática 104, North-Holland Publishing Co., Amsterdam, 1985.

[ 5 ] J. E. Gilbert, Maximal theorems for some orthogonal series I, Trans. Amer. Math. Soc. 145 (1969), 495515.

[6] D. L. GuY, Hankel multiplier transforms and weighted p-norms, Trans. Amer. Math. Soc. 95 (1960), $137-$ 189.

[ 7 ] Y. KAnJin, A transplantation theorem for Laguerre series, Tohoku Math. J. (2) 43 (1991), 537-555.

[ 8 ] Y. KAnJIN, On Hardy-type inequalities and Hankel transforms, Monatsh. Math. 127 (1999), 311-319.

[ 9 ] N. N. LebEDEv, Special functions and their applications, Dover Publications, Inc., New York, 1972

[10] A. MiYACHI, A transplantation theorem for Jacobi series in weighted Hardy spaces, Adv. Math. 184 (2004), 177-206.

[11] A. MiYACHI, Transplantation theorem for Jacobi series in weighted Hardy spaces, II, preprint.

[12] B. Muckenhoupt, Transplantation theorems and multipliers for Jacobi series, Mem. Amer. Math. Soc. 64 (1986), iv+86.

[13] S. SCHINDLER, Explicit integral transform proofs of some transplantation theorems for the Hankel transform, SIAM J. Math. Anal. 4 (1973), 367-384.

[14] E. M. Stein, Singular integrals and differentiabitity properties of functions, Princeton Mathematical Series 30, Princeton University Press, Princeton, N. J., 1970.

[15] G. N. WAtson, A treatise on the theory of Bessel functions, Cambridge University Press, London, 1966.

DePartment of Mechanical SyStems ENGineERING

FACULTY OF ENGINEERING

KANAZAWA UNIVERSITY

KANAZAWA 920-8667

JAPAN

E-mail address: kanjin@kenroku.kanazawa-u.ac.jp 